\title{
You Aren't Alone: Building Arabic Online Supporting Communities using Recommender System
}

\author{
Monirah Alajlan ${ }^{1}$, Nouf Alsuhaymi ${ }^{2}$, Sara Alnasser $^{3}$, Abeer Almohaidib ${ }^{4}$ \\ Nouf Bin Slimah ${ }^{5}$, Madawi Alruwaished ${ }^{6}$, Najla Alosaimi ${ }^{7}$ \\ Information Systems Department \\ King Saud University, Riyadh, Saudi Arabia
}

\begin{abstract}
People are now digitally connected, making the world a single large community. This remarkable benefit has solved many communication issues. For instance, people who go through difficult times and lack the emotional support required to overcome these crises can now join an online support group. For many years, such people had to travel to a predetermined location in a predetermined time to join a support group. Today, with the increasing availability of digital services, these groups can now meet online. For these reasons, this paper presents ' $\mathrm{You}$ aren't alone' mobile application, an interactive mobile-based application designed for Arab people who need psychological support. This application will help in enriching the Arabic content in the field of social support and will help in building supporting communities by peering users to the appropriate support group, anonymously without the fear of judgment. The application will enhance the peering process through a recommender system that reads the user's Twitter timeline and classifies the tweets as belonging to one of the available support groups.
\end{abstract}

Keywords-Emotional support; recommender system; classification; support group

\section{INTRODUCTION}

Over the years, technology has changed very rapidly resulting in the emergence of social media which has changed the way we communicate with one another. Currently, there is an extensive variety of social networking sites that create an environment where users can reach the maximum number of people.

Research has shown that the importance of social support is as significant as healthcare support. Recent studies have found that the availability and quality of emotional support have an essential role in prevention or even recovery of illnesses. In fact, the loss or even a lack of social support has been linked to various illnesses and conditions [1]. Recent research has focused on emphasizing the importance of social support networks in the area of communication technologies [2]. These include understating the importance of social support networks and their psychological effects and how we might apply such knowledge to design and develop relevant interventions.

On a local scale, the social support issue is alarming and the need for support groups is rising due to the increase in the prevalence of psychological issues which need support, such as patients with psychological disorders, diabetes mellitus, hypertension, bronchial asthma, and others. However, few studies have been conducted in Saudi Arabia to determine the prevalence of psychological issues among Saudi people. A study conducted by AlKahtani [3], measured the prevalence rate of psychological issues in a random sample of Saudi adults, aged from 15 to 65 years, which was found to be $18.2 \%$. Additionally, a study conducted by Al-Sughayr and Ferwana [4] covered a random sample of high school students and showed that the prevalence rate of psychological issues was $48 \%$. Therefore, this problem requires proper intervention in the form of social support. Unfortunately, to the best of our knowledge, literature review on Arabic applications revealed the fact that there is a lack of applications that provide psychological support and thus, this application will address this issue.

Considering the critical need for social support in Saudi Arabia, 'You aren't alone' application aims to fulfil the following objectives. First, to help and support the country's people and those of the Arab region in general who lack emotional support. Second, to include and support people who undergo similar issues regardless of their psychological nature. Such issues might be as simple as the struggles of new mothers, the confusion of fresh graduates, and the disappointment of unemployed graduates. Third, the application aims to build a network of people who have similar issues, in order to share and view thoughts, stories, and experiences. Moreover, they can create their own virtual reading rooms and ask questions, and life coaches and experts may volunteer to help and answer questions. Furthermore, the application aims to enhance the process of peering the users with the appropriate supporting group through Twitter data mining.

The application can suggest a support group for the user by linking their account to Twitter. As nowadays Twitter is considered as an expressive platform where people's thoughts and emotions can be reflected by their tweets. By using Twitter's API to stream user's tweets and then applying topic modeling techniques to classify tweets into their relevant support group. Twitter is an online microblogging tool that disseminates more than 400 million messages per day [5]. As the tweets allow to gain an insight into the online public behavior, it represents an important data source to conduct textual analysis. Twitter is considered more effective than other social media platforms for analysis because the tweets are publicly available and easily accessible via APIs [6].

This paper is structured as follows. Section II illustrates the literature review that discusses related studies and applications. 
A comprehensive comparison against similar systems is shown in Section III. Section IV presents the application development process used in the project. Section $\mathrm{V}$ discusses the limitations and future work, and Section VI concludes the paper.

\section{LITERATURE REVIEW}

This section begins with a literature review to present previous studies that discussed topics related to the concept of mental health. Next, a comparison of similar applications based on differing features is presented. Finally, we present a discussion that summarizes the results and outcomes.

\section{A. Related Studies}

Life can be hard for many people. Unfortunately, a lot of people who suffer from issues that are triggered by life's events such as loss of loved ones, family-related issues, isolation, and many more end up with psychological distress. A study in [7] revealed that individuals with low connectedness exhibit more dysfunctional interpersonal behaviors which in turn contribute to more psychological distress. Similarly, individuals with high connectedness exhibit more appropriate interpersonal behaviors which in turn contribute to less psychological distress [7]. In the same vein, a study in [8] investigated the effectiveness of joining support groups for patients with contact dermatitis, reported that $77.8 \%$ of the patients noticed a positive difference in the way they understand and cope with this illness. This positive effect was also reported in [9], where the COVID-19 pandemic has caused enormous stress on people working in the health care sector. The study concluded that joining a virtual support group was indeed helpful during the crisis.

Another problem with social support is that some people find it embarrassing to reveal their identity in a setting where they express their deepest emotions and pain. This might be because people are usually afraid of other people's judgment. Research indicates that even experts often judge others and that the diagnostic judgments made by counselors are often influenced by their preferences for different types of counseling problems and their biases in the use of diagnostic classification systems. Studies have proven that the final number of counseling interviews is affected by the type of the client's issue and certain of their descriptive characteristics [10].

By conceptualizing social networking platforms as new social domains, online social support could supplement the mental health benefits derived from in-person social support [11]. Research suggests that people who are more introverted or socially anxious might be more likely than others to derive benefit from social media. Particularly, there is an absence of supportive apps that can help people who need emotional support and reduce their sense of loneliness in the Arab world [9]. To the best of our knowledge, our search in Android and Apple markets yielded no apps dedicated to this issue.

Twitter has increasingly been used by individuals to express their thoughts, and feelings in the form of short text messages. In their 2014 study [12], Hasan et al. concluded that classifying short texts according to finer-grained classes of emotions provides rich and informative data about the emotional states of individuals. In addition, these data can be used by healthcare professionals for early detection of psychological disorders such as anxiety or depression.

\section{B. Related Applications \\ - Sanvello}

Sanvello is a website and a mobile app for both iOS and android [13]. It aims to help people suffering from stress, anxiety, and depression and it offers several tools to address mental health issues. These tools are based on cognitive behavior therapy, mindfulness meditation, relaxation, and $\mathrm{mood} /$ health tracking. Users can go by a nickname when they share their stories, advice, and more with Sanvello's peer support community via a specified topic discussion or a chat group. The application provides special features such as when it is first launched, the user will get a message telling him "take a moment to pause and think of a place that relaxes you". Subsequently, the user will choose a theme, set goals, and build a daily habit of self-care.

\section{- Lyf}

Lyf is a social media app where users share their feelings and experiences by posting, connecting, and chatting with other users who share the same concern [14]. In the "Feed", users can post their thoughts in a timeline. It illustrates the "stream" in which users share their journeys as private, public, or anonymous to share stories sequentially and to keep updating on their story. Users can also chat with other people with the same concern or with "live support" which is an online counselling platform for users who seek professional advice and guidance.

\section{- We are close}

It is an official android and iOS application introduced by the Saudi Ministry of Health [15]. This application allows members to read topics and articles related to mental health with the aim to educate people about mental health issues. Interestingly, members can consult with specialists by filling a form with information such as the age, gender, the problem for consulting, medications they use, and any other health problems. The application also has a section for voice messages to communicate directly with the health specialists. Additionally, there is a section of interaction in which the member can add an article or a picture or write a motivational story. There are books that tackle mental health issues that have been made available to users on the application. The benefit of this application is that users do not have to register first to use the application; they can take a look and use some features as guest.

\section{- ReachOut}

ReachOut is to support network for patients and care givers. Essentially, it is an iOS and android application but has a blog dedicated to inspiring, educating, and connecting readers with real-world health stories and articles. It is a peer support app categorized into seven categories for mental health, cancer, diabetes, chronic pain, etc. Users can choose more than one category. The primary interaction is through a timeline where users share their thoughts and others can "give a hug" or write a comment. Users also can communicate with 
each other through messages which facilitate private communication supporting and sharing more feelings. To use the application, first one has to create an account and then manage their profile by filling the name, age, status, and gender; subsequently, one will be able to use the application.

\section{COMPARISON WITH RELATED APPLICATIONS}

Comparing similar applications in a table demonstrates the differences between the applications easily. Table I illustrates the five applications including our application and compares them in terms of multiple features including functionality, platforms, and the content. It can be seen that 'You aren't alone' has several of the important features that exist in similar applications, such as the timeline and anonymity. On the other hand, it provides features that are not supported in any other applications like suggestion based on tweets.

TABLE I. COMPARISON WITH SIMILAR SYSTEMS

\begin{tabular}{|c|c|c|c|c|c|}
\hline Features\App & Pacifica & Lyf & $\begin{array}{l}\text { We are } \\
\text { close }\end{array}$ & ReachOut & $\begin{array}{l}\text { You Aren't } \\
\text { Alone }\end{array}$ \\
\hline Timeline & $\checkmark$ & $\checkmark$ & $\checkmark$ & $\checkmark$ & $\checkmark$ \\
\hline Anonymous & $\checkmark$ & $\checkmark$ & $\checkmark$ & $x$ & $\checkmark$ \\
\hline Likes & $\checkmark$ & $x$ & $\checkmark$ & $\checkmark$ & $\checkmark$ \\
\hline $\begin{array}{l}\text { Join specific } \\
\text { support group }\end{array}$ & $\checkmark$ & $x$ & $x$ & $\checkmark$ & $\checkmark$ \\
\hline $\begin{array}{l}\text { Create a } \\
\text { support group }\end{array}$ & $x$ & $x$ & $x$ & $x$ & $\checkmark$ \\
\hline $\begin{array}{l}\text { Participation } \\
\text { of Therapist } \\
\text { and life } \\
\text { coaches }\end{array}$ & $x$ & $\checkmark$ & $\checkmark$ & $x$ & $\checkmark$ \\
\hline Share articles & $x$ & $x$ & $\checkmark$ & $\checkmark$ & $\checkmark$ \\
\hline $\begin{array}{l}\text { Suggest group } \\
\text { based on } \\
\text { twitter }\end{array}$ & $x$ & $x$ & $x$ & $x$ & $\checkmark$ \\
\hline $\begin{array}{l}\text { Question and } \\
\text { Answers }\end{array}$ & $x$ & $x$ & $\checkmark$ & $x$ & $\checkmark$ \\
\hline $\begin{array}{l}\text { Subscription } \\
\text { fees for more } \\
\text { features }\end{array}$ & $\checkmark$ & $\checkmark$ & $\checkmark$ & $\checkmark$ & $x$ \\
\hline Website & $\checkmark$ & $x$ & $x$ & $\checkmark$ & $x$ \\
\hline $\begin{array}{l}\text { Mobile } \\
\text { Application }\end{array}$ & $\checkmark$ & $\checkmark$ & $\checkmark$ & $\checkmark$ & $\checkmark$ \\
\hline Arabic & $x$ & $x$ & $\checkmark$ & $x$ & $\checkmark$ \\
\hline
\end{tabular}

\section{APPLICATION DEVELOPMENT}

\section{A. Information Gathering}

1) Interview: The interview is one of the most important techniques for information gathering; it helps collect a richer source of information on behavior, opinions, knowledge, and preferences. Therefore, three experts were selected to gain insights from their experiences.

Several interviews were conducted with specialists in the field of psychology. A profound physician stated that psychological support is a psychological need for each human being. He explains the concept of Group Psychotherapy as where people share their experience, symptoms and experience in treatment. It's a codified type of psychological treatment.

Effective communication reduces the symptoms of psychological illness. Also, effective communication has a chemical effect also, it changes the proportion of chemicals in the body that affect the receptors of those chemicals in the body.

Furthermore, a college professor confirmed for us the importance of psychological support and considered it the basis for maintaining good mental health. When asked about the importance of sharing similar experiences, she said that research in developed countries has revealed that people should engage in psychosocial sessions because sharing feelings with others helps to alleviate the feelings but locking it up can turn it from a problem situation into a state of depression, nervousness or tension and then social isolation which may affects the person negatively. In the same vein, a lecturer stressed the importance of supporting the individual first and the society second. An individual is the basic building block of society and by supporting him we might create a healthy society with awareness and freedom. Moreover, sharing feelings with others might facilitate social interaction and emotional support. However, sometimes people have difficulty sharing feelings because they may expect no reaction to their feelings or may have been shy since childhood. Unfortunately, not sharing tragedies or personal issues might lead to several behavioral problems such as social shyness and lack of selfconfidence, which affects productivity.

2) Questionnaire: Based on a study covering a sample of 937 people aged 14 and above, we found out that native Arabic speakers are facing issues with the availability of resources in social and emotional support as more than $50 \%$ of the people questioned agreed that there is a lack of Arabic content for psychological and social support platforms. For instance, $53.7 \%$ of the sample have faced difficult times and experiences and sought support with no luck, while others wanted to contact life coaches and therapists but never got the opportunity. We also found out that a significant percentage of those who were questioned are feeling a lot of social pressure as $54 \%$ of the respondents wanted to remain anonymous while expressing their true feelings, and more than $50 \%$ of the respondents felt that they fear judgments, blame, and misunderstanding when sharing personal stories. On the other hand, we found that there are some improvement mechanisms 
that motivate people to connect and share their feelings. For instance, most respondents stated that they prefer talking to people with similar personal experiences as such people understand them which makes them feel better. Interestingly, most respondents stated that social support improves confidence and sense of self-fulfillment, and $77 \%$ of respondents indicated that volunteering and community service improves their psychological wellbeing. Another $84 \%$ were willing to provide help to those in need for emotional and psychological support. We also found that people care about sharing valuable news and research papers as $90 \%$ feel better when friends share articles and news that matters to them. When asked about their feelings about developing an application that provides psychological health care and support groups in Arabic, more than $96 \%$ of respondents chose the option "great idea". Based on the aforementioned results and due to the lack of available resources in social support and dearth of Arabic content, we aim to provide a social support platform in Arabic to help Arab communities that need emotional support share their experiences and relieve all the ambiguous emotions they might be feeling by connecting them with a group of people with similar experiences along with the supervision of therapists and life coaches who would love to join voluntarily.

\section{B. System Implementation}

You aren't alone system is composed of three main components. The first one is the application program which is developed using Xcode IDE platform that supports iOS application development with Swift programming language. The Interface Builder within Xcode is used to design the user interface. The second component is Firebase Real-time database which is responsible for storing retrieving and manipulating system's data. Firebase real-time database is a cloud-hosted database which is a collection of objects stored in a tree-like hierarchical structure. Fig. 1 illustrates the database collections.

The third component is the data mining component, where a user's tweets are mined in order to produce suitable support groups. Twitter is an online microblogging tool that disseminates more than 400 million messages per day [5]. As the tweets allow to gain an insight into the online public behavior, it represents an important data source to conduct textual analysis. The "Suggest for me" function goes through several phases as shown in Fig. 2.

1) Data collection: Twitter Kit is a native SDK to include Twitter content in mobile apps [16]. It provides many features like displaying Tweets, authorizing Twitter users, and working with the Twitter API. First, using "Log in With Twitter" feature provided by Twitter kit to enables app users to authenticate with Twitter. Then building a query to retrieve random tweets from the public timeline. A dataset of over 500 tweets was collected and stored in a .csv file.

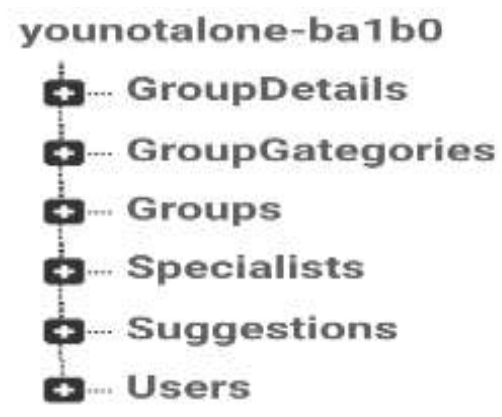

Fig. 1. Firebase Database Collection.

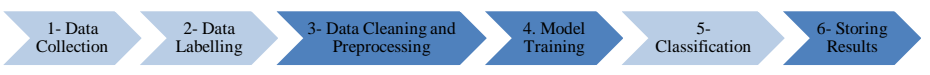

Fig. 2. 'Suggest for Me' Phases.

2) Dataset labelling: After collecting tweets from the public timeline, manual labelling by the team was done, in order to classify each tweet as related to one of the ten support groups available such as depression and diabetes.

3) Data cleaning and pre-processing: Data go through two steps cleaning and pre-processing. In cleaning, the punctuation, stop words, and URLs are removed because they carry little meaning. Whereas in pre-processing, the text is normalized to scale the data into fewer ranges.

In the case of suggesting support groups based on the users' tweets, it was recognized that there are some words that carry more meaning than other words. For instance, the world 'depression' is more valuable than the world 'having'. In order to eliminate the worthless words before it takes valuable processing time, NLTK was use. The NLTK corpus library detect and extract the commonly used stop words. For example, in this tweet: "I need to improve myself to save my relationship." After running the code using NLTK to detect and remove the stop words the output was: ['need', 'improve', 'myself', 'save', 'relationship'].

4) Model training: In this step, one of the Natural Language Processing techniques, the topic modelling is used. Topic modelling is a process to automatically identify topics present in a text object and to derive hidden patterns exhibited by a text corpus [17]. An example of topic modelling algorithm is Latent Dirichlet Allocation (LDA), it is used to discover themes in text and classify text into those predetermined themes.

Using LDA topic modeling to analyze the tweets and their related support groups, a dictionary of keywords for each support group is created. This is accomplished through calculating the probabilities of words and deciding which of them are closely related to the topic of the support group. The dictionary is divided into 10 categories, each of them represents a group and contains words that reflect the emotion related to the group. Table II shows sample terms from the created dictionary. 
TABLE II. SAMPLE TERMS FROM THE DATASET

\begin{tabular}{|c|c|c|c|c|c|c|c|c|c|}
\hline 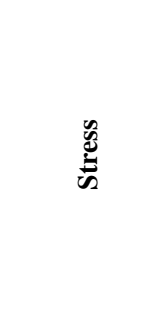 & 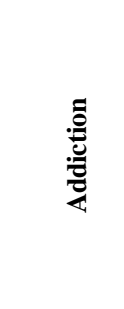 & 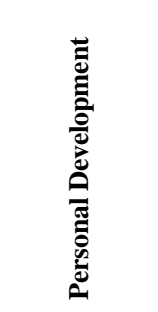 & 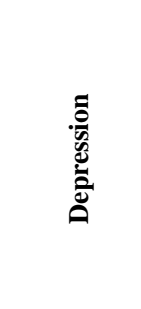 & 吾 & 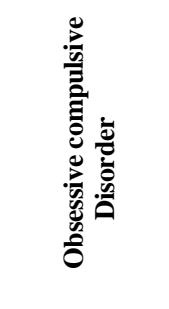 & 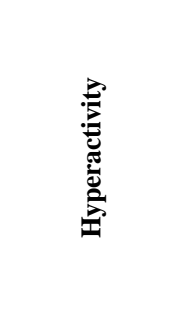 & 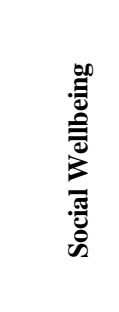 & 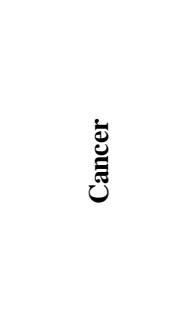 & 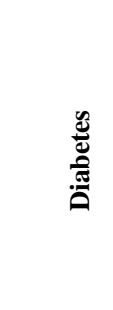 \\
\hline Overthinking & Addiction & Self esteem & Feel lonely & Diet & OCD & ADHD & Fight & Chemotherapy & Diabetes \\
\hline Stressed out & Smoke & Self identify & Sad & Health & $\begin{array}{l}\text { Obsessive } \\
\text { compulsive } \\
\text { disorder }\end{array}$ & Hyperactivity & Hate & Leukemia & TID \\
\hline Stress & Drug & Insecurity & Worried & Weight & Compulsions & Hyper & Rude & Breast cancer & $\mathrm{T} 2 \mathrm{D}$ \\
\hline anxious & cigarettes & confident & depressed & food & obsessions & $\begin{array}{l}\text { Extreme } \\
\text { hyperactivity }\end{array}$ & problems & Lung cancer & insulin \\
\hline
\end{tabular}

5) Classification: In this phase, the system mainly works with an end-point API to preform NLP classification. Firstly, using LDA topic classifier to analyze the user profile, specifically the last 10 tweets on his timeline. In order to return group suggestions to the user given the last ten tweets, the model calculates and tests the probabilities for each class resembling the group topic and compares them to the stored key words from the initial training dataset. Then, it returns a list of support groups with the highest probabilities based on the tweets' text. In this case, the results contain the suggested group, confidence and the reason.

6) Storing results: Once the analysis gets the appropriate groups for the user, it's then automatically added to the user joined groups. As it is a one-time process there is no need to store the analysis result nor the user twitter information.

\section{System Design}

For 'You aren't alone' application, the most suitable architecture is a combination of Model View Presenter (MVP) and client server architectures as shown in Fig. 3. Client-server architecture can be defined as a software architecture made up of both the client and server, whereby the clients always send requests while the server responds to the requests sent [18].

Since the application, as a client, requires accessing several servers such as Firebase and Twitter API in order to establish a connection and send requests, the client-server approach was a logical solution [19]. Furthermore, MVP allows for decoupling code in presenter level and by that enabling reuse of the code interface of the application without the need of using third party tools.

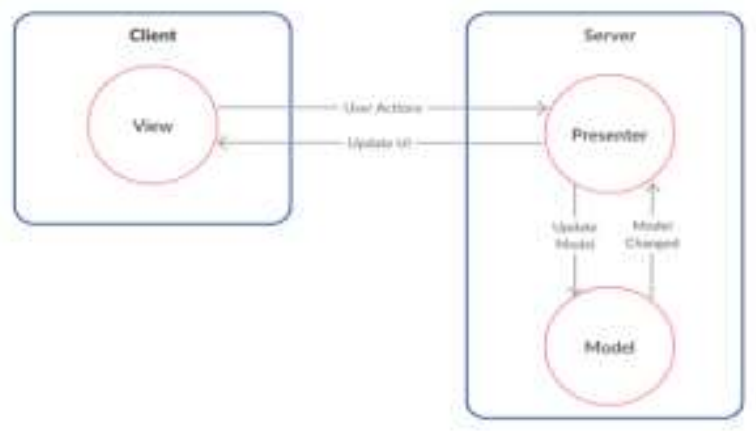

Fig. 3. You Aren't Alone Architecture.

\section{User Interface}

This section presents a sample of screens in You aren't alone to illustrate the user interface design (Fig. 4 to 7).

\section{E. System Evaluation and Testing}

1) Acceptance testing: User Acceptance Testing (UAT) plays an important role in validating the requirement by target users to prevent losses after releasing the system. UAT was conducted with users from different ages and different concerns to make sure the system provides a variety of support groups.

Two stages of testing were conducted. The first stage is to calculate the time each user spent on each task. The second stage is a survey on the same participants with 7 questions regarding system's functionality, clearance, user-friendliness, interfaces. Table III displays a sample of the user acceptance testing. Based on the results the users took much longer time in posting a thought and asking questions. It takes longer for the users to type than to view as well as expressing their feelings might need a longer time. 


$$
\text { 圆首 }
$$


TABLE III. ACCEPTANCE TESTING RESUlts

\begin{tabular}{|c|c|c|c|c|}
\hline \multirow{2}{*}{$\begin{array}{l}\text { Tester name } \\
\text { Task }\end{array}$} & \multicolumn{4}{|c|}{ Haifa Majed (Member) } \\
\hline & Number of errors & Time needed & User's feedback & Completion status \\
\hline Browsing home page & 0 & 00:00:36 sec & - & Completed. \\
\hline Linking to twitter. & 0 & 00:00:11 sec & "Great characteristic" & Completed. \\
\hline Suggest a support group & 0 & 00:00:42 sec & - & Completed. \\
\hline Browsing support groups. & 0 & 00:00:08 sec & - & Completed. \\
\hline Posting a thought & 0 & 00:00:46 sec & - & Completed. \\
\hline Check the timeline & 0 & 00:00:07 sec & "Presented very well" & Completed. \\
\hline Reply a thought & 0 & 00:00:21 sec & - & Completed. \\
\hline Share articles & 0 & 00:00:22 sec & - & Completed. \\
\hline Pin articles & 0 & 00:00:04 sec & - & Completed. \\
\hline Ask questions & 0 & 00:00:30 sec & - & Completed. \\
\hline Answer to questions & 0 & 00:00:16 sec & - & Completed. \\
\hline Browse the whole app & 0 & 00:04:05 min & - & Completed. \\
\hline
\end{tabular}

\section{LIMITATIONS AND FUTURE WORK}

One of the limitations of the system would be that it is only available for iOS users and due to this limitation, we intend to extend the scope of the system to support a Web application platform and Android devices. Another limitation is that it only extracts textual features form Twitter using text mining. However, deep emotions are sometimes hidden in the images a user post.

The system currently supports Arabic language only, but we plan to expand our system to include universal languages (ex. English). Another future direction would be to improve the recommendation process by, expanding the scope of the system to generate recommendations of support groups based on user past behaviors rather than the content of users' tweets. Past behavior includes the browsing habits and frequently used website since they give a strong indication of his psychological state and/or mood.

\section{CONCLUSION}

In this paper, 'you aren't alone' application, an interactive mobile application dedicated for those who need social support in the form of support groups, was introduced. Although people are becoming more aware about the psychological issues, many problems arise when a person wants to join a support group, such as the lack of confidence and the fear of judgment. The application aims towards advancing the mental and psychological health of people by providing adequate support groups. Despite the numerous applications in this field, 'you aren't alone' is one of a few applications that targets the Arab community and the first to suggest a support group based on a user's tweets. The team hope that 'You aren't alone' becomes a supportive and inviting environment where people struggling in life, find it a safe place to freely express their feelings and engage with others.

\section{ACKNOWLEDGMENT}

This research project was supported by a grant from the "Research Center of the Female Scientific and Medical Colleges", Deanship of Scientific Research, King Saud University.

\section{REFERENCES}

[1] Marilyn Frank-Stromborg, Sharon J. Olsen. "Instruments for clinical Health-care Research”. Dec 2004.

[2] Heather Attig. "Social support and new communication technologies during a life stressor". Nov 2013.

[3] Al-Khathami AD, Ogbeide DO. "Prevalence of mental illness among Saudi adult primary-care patients in Central Saudi Arabia". Saudi Med J., Jun 2002.

[4] Al-Sughayr AM, Ferwana MS." Prevalence of mental disorders among high school students in National Guard Housing, Riyadh, Saudi Arabia”. J Fam Community Med, Jan 2012.

[5] A. Kim, J. Murphy, J. Duke, H. Hansen, A. Richards, and J. Allen. "Methodological Considerations in Analyzing Twitter Data". Journal of the National Cancer Institute. Monographs. 2013.

[6] Van den Rul, C. (2019). A Guide to Mining and Analysing Tweets with R. Medium. Available at: https://towardsdatascience.com/a-guide-tomining-and-analysing-tweets-with-r-2f56818fdd16. [Accessed online on 9/12/2019].

[7] Richard M., Matthew Draper and Sujin Lee. "Social Connectedness, Dysfunctional Interpersonal Behaviors, and Psychological Distress: Testing a Mediator Model", Journal of Counseling Psychology. Vol. 48, No. 3, 310-318, 2011.

[8] L. Voller, S. Kullberg, Y. Liou and S. Hylwa, "Effectiveness of Support Groups for Patients With Contact Dermatitis: A Pilot Study", Dermatitis, vol. 31 , no. 6 , pp. 383-388, 2020. 
[9] Viswanathan, M. Myers and A. Fanous, "Support Groups and Individual Mental Health Care via Video Conferencing for Frontline Clinicians During the COVID-19 Pandemic", Psychosomatics, vol. 61, no. 5, pp. 538-543, 2020.

[10] John B., Richard S., Deborah M. "Counselor Intake Judgments, Client Characteristics, and Number of Sessions at a University Counseling Center", Journal of Counseling Psychology.975, Vol. 22, No. 6, 567559.

[11] David A., Elizabeth A., Rachel L., Kathryn M., and Tawny Spinelli. "Online Social Support for Young People: Does It Recapitulate Inperson Social Support; Can It Help?". Dec 2016.

[12] M. Hasan, E. Rundensteiner, and E. Agu, "EMOTEX: Detecting Emotions in Twitter Messages" 2014 ASE bigdata/socialcom/cybersecurity Conference. Available at: http://web.cs.wpi.edu/ emmanuel/publications/PDFs/C30.pdf.

[13] Sanvello A Place to Feel Better, https://www.sanvello.com/, [Accessed November 29, 2020].
[14] Lyf. Lyf App Ltd, 2020 [Online]. Available: https://play.google.com/store/apps/details?id=com.incogo.android\&hl=e $\mathrm{n} \& \mathrm{gl}=\mathrm{US}$, [Accessed November 21, 2020].

[15] We are close: Your own Clinic at Home, Available: http://ncmh.org.sa/index.php/pages/view/90/14/14, [Accessed November 28, 2020].

[16] Tools and Libraries, Twitter Inc, [online]. Available: https://developer.twitter.com/en/docs/tools-and-libraries.

[17] Beginners Guide to Topic Modeling in Python and Feature Selection. Analytics Vidhya. Available https://www.analyticsvidhya.com/blog/2016/08/beginners-guide-totopic-modeling-in-python/. [Accessed online on 10/12/2019].

[18] H. Oluwatosin. "Client-Server Model", IOSR Journal of Computer Engineering, vol. 16, no. 1, pp. 57-71, 2014.

[19] V. Corneliu, M.Iulian, Octavian. "Model View Presenter Design Pattern", Journal of Computer Science and Control Systems. vol. 3, pp. 173-176, 2010. 